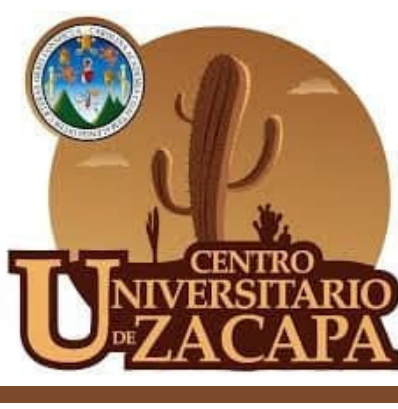

Recibido: 31/08/2021

Artículo científico

Aceptado: 20/01/2022

\title{
Infodemia, como factor desencadenante del miedo al COVID-19
}

\author{
Infodemic, as a trigger for fear of COVID-19
}

Luz Andrea Castañeda Argueta

Maestría en Neurociencias con énfasis en Neurocognición

Universidad San Carlos de Guatemala

luzandreacastaneda.11@gmail.com

https://orcid.org/0000-0002-7538-1181

\section{Referencia del artículo}

Castañeda Argueta, L. A. (2022). Infodemia, como factor desencadenante del miedo al COVID-19. Revista Académica CUNZAC. 5(1), 33-40. DOI: https://doi.org/10.46780/cunzac.v5i1.54

\section{Resumen}

OBJETIVO: describir la presencia de miedo al coronavirus (COVID-19) desencadenado por la infodemia en profesionales de la salud mental. MÉTODO: estudio descriptivo de corte transversal, con una muestra representativa de 303 profesionales. RESULTADOS: se determinó que el rango etario prevaleciente en la población de estudio estuvo comprendido entre 18 a 35 años con un $60.39 \%$, el género predominante fue el femenino con un $82.83 \%$, la mayoría de los profesionales indicaron estar solteros, obteniendo un $56.76 \%$. En lugar de residencia, se enfatizó en los departamentos de Guatemala, logrando mayor participación en Quetzaltenango con 28.05\%, Jutiapa con 18.81\%, Zacapa 17.82\%, Guatemala 17.16\% y Totonicapán con 3.63\%. La Activación Somática con un $89.10 \%$, fue el componente del miedo con mayor presencia en la población. CONCLUSIÓN: el $90 \%$ de los profesionales tuvo una puntuación representativa del miedo al COVID-19 igual o mayor a 17.5, resaltando que el $68.31 \%$ manifestó utilizar las redes sociales como medio de divulgación que más frecuentan para mantenerse informados del coronavirus, identificando Facebook con un 72.27 \%, como la red social más utilizada.

Palabras clave: infodemia, sobreinformación, miedo, pandemia, covid-19

\begin{abstract}
OBJECTIVE: describe the presence of fear of the coronavirus (COVID-19) triggered by the infodemic in mental health professionals. METHOD: descriptive cross-sectional study, with a representative sample of 303 professionals. RESULTS: it was determined that the prevailing age range in the study population was between 18 to 35 years old with $60.39 \%$, the predominant gender was female with $82.83 \%$, most of the professionals indicated being single, obtaining $56.76 \%$. Instead of residence, it was emphasized in the departments of Guatemala, achieving greater participation in Quetzaltenango with $28.05 \%$, Jutiapa with $18.81 \%$, Zacapa $17.82 \%$, Guatemala $17.16 \%$ and Totonicapán with $3.63 \%$. Somatic Activation with $89.10 \%$, was the component of fear with the greatest presence in the population. CONCLUSION: $90 \%$ of the professionals had a representative score of fear of COVID-19 equal to or greater than 17.5 , highlighting that $68.31 \%$ stated that they use social networks as a means of dissemination that they frequent the most to stay informed of the coronavirus, identifying Facebook with $72.27 \%$, as the most used social network.
\end{abstract}

Keywords: infodemic, overinformation, fear, pandemic, covid-19 


\section{Introducción}

Desde la aparición de los primeros casos por coronavirus (COVID-19) en Wuhan, China, se ha visto el desarrollo tan veloz de esta pandemia, que ha puesto en peligro los sistemas sociales, económicos y sanitarios (García-Marín, 2020), a su vez, se ha presenciado una gran cantidad de información falsa, compartida y mal interpretada por los medios de divulgación, alarmando a la población con publicaciones alteradas e impactantes, causando según Fernández Llontop, C. F. (2021) temor y agitación en los lectores.

Aleixandre-Benavent, R. y colaborados (2020), refieren que, lo mismo que ha sucedido en años anteriores con epidemias como el virus del ébola y el zika, se manifiesta en la actualidad con aspectos relacionados a la pandemia COVID-19.

Los medios de comunicación social y divulgación de información han aprovechado esta situación para dedicar secciones completas al coronavirus, sin tomar en cuenta la veracidad de la información y la manera en que la comparten al público, interviniendo en el desencadenamiento de emociones negativas, en las personas que se dejan llevar por noticias sin fundamento científico.

Una de las principales emociones que ha ocasionado está situación, es la manifestación del miedo al COVID-19 (Huarcaya-Victoria, et al., 2020), pues el solo hecho de pensar que se transmite de manera rápida e inevitable provoca temor (Vera, 2020) agregándole ahora, la mala interpretación de la información que se comparte referente al tema.

El miedo, es una de las emociones básicas, catalogada como la reacción más natural en situaciones amenazantes (Quezada, V. E., 2020), y si no se controla ante la ola de desinformación ocasionada por el coronavirus, puede llegar a generar angustia y mayor sensibilidad a la ansiedad, limitando la capacidad que se tiene para manejar estrategias adecuadas y poder regular las emociones, dificultando el afrontamiento de la pandemia.

Un claro ejemplo asociado a este tema, fue abordado por Mejía C. R. y colaboradores (2020), quienes hacen referencia de lo ocurrido en el año 2018 en Brasil, durante una campaña de vacunación contra la fiebre amarilla, cuando se compartió en redes sociales rumores de que la vacuna era mortal, activando el miedo en las personas y afectando la meta que se tenía programada, descendiendo de un $95 \%$ a $76 \%$ de la población vacunada.

La finalidad de la infodemia, no es reemplazar una información por otra, sino aumentar la duda y desconfianza en las personas, de manera que todas las noticias o relatos compartidos por los medios de divulgación parezcan sospechosos, situación que resulta muy perjudicial en relación a los avancen de la COVID-19 (García-Marín, 2020).

En abril del año 2020 "la base de datos CoronaVirus Fact Alliance, impulsada por el Poynter Institute y la International Fact-Checking Network, registró 3,800 engaños relacionados con el coronavirus circulando por todo el mundo" (Aleixandre-Benavent, et al., 2020) indicando que la COVID-19 ha desencadenado un sinfín de rumores que han provocado la desconfianza en

Volumen 5 | Número 1 |Enero - Junio 2022 
las medidas de prevención, las indicaciones médicas, las compras desmedidas y la aplicación de la vacuna, perjudicando la salud y aumentando los contagios.

Resaltando este impacto de la infodemia en la población en general, también han empezado a surgir inquietudes respecto al afrontamiento de los profesionales que acompañan a las personas en estas situaciones, quienes, a pesar de su formación académica, no son absueltos de ser víctimas de la sobreinformación.

En un estudio realizado por Lozano-Vargas (2020), enfocado en el impacto que provoca la COVID-19 en la salud mental del personal de salud en China, resalta que estos profesionales también pueden experimentar: estrés, ansiedad, síntomas depresivos, insomnio, negación e inestabilidad emocional, y que a la vez están propensos al agotamiento, angustia moral vinculada a la experiencia personal o familiar de COVID-19 y miedo cuando se está en presencia de desconocidos que pueden portar el virus.

Por lo que, el reciente estudio, tiene como objetivo describir la presencia del miedo al coronavirus (COVID-19), desencadenado por la infodemia en profesionales de la salud mental. Resaltando que en un periodo en el que muchas entidades importantes trabajan en conjunto y se esfuerzan por combatir el coronavirus, la influencia de la infodemia en el desencadenamiento de emociones negativas durante la pandemia COVID-19, también debe ser tomado en cuenta, sin dejar fuera a nadie, porque puede ser una clave importante para identificar y disminuir la propagación de signos y síntomas relacionados con padecimientos psicológicos durante el confinamiento, en los lectores que no ratifican si la información o medios de divulgación que frecuentan tienen fundamento científico.

\section{Materiales y métodos}

Se realizó un estudio descriptivo de corte transversal, con una muestra representativa de 303 profesionales de la salud mental y miembros suscritos a un grupo de una red social, conformado por psicólogos de toda la república de Guatemala.

La presentación y análisis de resultados, se ostentó por medio de tablas, estructuradas con información obtenida de la boleta de recolección de datos y su tabulación a través del programa Excel, conformada por características sociodemográficas, preguntas enfocadas en la infodemia, componentes del miedo y la Scale Fear of COVID-19 (FCV-19S), por sus siglas en inglés. Se hizo uso de la versión en español "Escala de Miedo al Coronavirus (COVID-19)" (Huarcaya-Victoria, et al., 2020), adaptada al contexto ecuatoriano, utilizada en el primer estudio metodológico enfocado en este tema por Ramírez-Coronel, A. A., y colaboradores (2020). 


\section{Resultados}

Tabla 1. Medios de divulgación y redes sociales que más frecuentan los profesionales de la salud mental.

\begin{tabular}{|c|c|c|}
\hline Medios de divulgación & Cantidades & Porcentajes \\
\hline 1. Redes sociales & 207 & $68.31 \%$ \\
\hline 2. Medios electrónicos & 61 & $20.13 \%$ \\
\hline 3. Medios de divulgación científica & 27 & $8.91 \%$ \\
\hline 4. Medios impresos & 08 & $2.64 \%$ \\
\hline \multicolumn{3}{|l|}{ Redes sociales } \\
\hline 1. Facebook & 219 & $72.27 \%$ \\
\hline 2. Otros & 34 & $11.22 \%$ \\
\hline 3. WhatsApp & 16 & $5.28 \%$ \\
\hline 4. Twitter & 14 & $4.62 \%$ \\
\hline 5. Instagram & 13 & $4.29 \%$ \\
\hline 6. YouTube & 07 & $2.31 \%$ \\
\hline
\end{tabular}

Fuente: elaboración propia, boleta de recolección de datos con base a los medios de divulgación y redes sociales.

Mediante el análisis de resultados, los medios de divulgación que más frecuentan los profesionales, enumerados según los porcentajes obtenidos son: 1. redes sociales con un 68.31 \%, 2. medios electrónicos con $20.13 \%$, 3. medios de divulgación científica con $8.91 \%$ y 4. medios impresos con $2.64 \%$ posicionando en primer lugar a las redes sociales. También se identifica que la red social que utilizan con más frecuencia la población de estudio es Facebook con un 72.27 \%.

Tabla 2. Componentes del miedo que presenta la población de estudio.

\begin{tabular}{lcc}
\hline Componentes del miedo & Cantidades & Porcentajes \\
\hline Activación somática & $\mathbf{2 7 0}$ & $\mathbf{8 9 . 1 0} \%$ \\
\hline Preocupación & 211 & $69.63 \%$ \\
\hline Ansiedad & 32 & $10.56 \%$ \\
\hline Nerviosismo & 14 & $4.62 \%$ \\
\hline Tensión & 13 & $4.29 \%$ \\
\hline Proceso cognitivo & $\mathbf{1 7 8}$ & $\mathbf{5 8 . 7 4} \%$ \\
\hline Temor a las manifestaciones físicas & 123 & $40.59 \%$ \\
\hline Anticipación a consecuencias negativas & 39 & $12.87 \%$ \\
\hline Sentimiento de valoración negativo & 10 & $3.30 \%$ \\
\hline Descalificación Personal & 06 & $1.98 \%$ \\
\hline Manifestaciones conductuales & $\mathbf{1 6 5}$ & $\mathbf{5 4 . 4 5} \%$ \\
\hline Bloqueo Mental & 112 & $36.96 \%$ \\
\hline Sudoración & 33 & $10.89 \%$ \\
\hline Presentación de tics & 18 & $5.94 \%$ \\
\hline Temblor en la voz & 02 & $0.66 \%$ \\
\hline
\end{tabular}

Fuente: elaboración propia, boleta de recolección de datos con base a los componentes del miedo.

Volumen 5 | Número 1 |Enero - Junio 2022 
De acuerdo a los porcentajes, los componentes del miedo que se presentan en la población de estudio son: Activación Somática con un 89.10 \%, resaltando la preocupación como característica principal de este componente. El Proceso Cognitivo, es posicionado como el segundo componente con un 58.74 \% dejando por ultimo a las Manifestaciones Conductuales con un $54.45 \%$.

Tabla 3. Miedo al coronavirus (COVID-19) presentado en profesionales de la salud mental, según la escala FCV- $19 S$.

\begin{tabular}{lcc}
\hline $\begin{array}{l}\text { Puntuación representativa del miedo } \\
\text { al CoVID- 19 }\end{array}$ & Cantidades & Porcentajes \\
\hline Mayor a 17.5 & 145 & $47.85 \%$ \\
\hline Menor a 17.5 & 141 & $46.53 \%$ \\
\hline Igual a 17.5 & 17 & $5.61 \%$ \\
\hline
\end{tabular}

Fuente: elaboración Propia, boleta de recolección de datos con base a la escala FCV-19S.

En la tabla 3 se ostentan los datos obtenidos a través de la escala (FCV-19S), divididos en tres grupos (mayor a 17.5, igual a 17.5 y menor a 17.5) partiendo de las puntuaciones totales, mismas que oscilan entre 7 y 35 puntos, tomando como base la media de la puntuación mayor (35), con la intención de establecer puntuaciones que permitieran manifestar de una forma más comprensible el miedo presentando por la población de estudio. Se comprobó la detección de miedo al COVID-19 en profesionales de la salud mental, resaltando que el $47.85 \%$ de la población, obtuvo una puntuación representativa mayor a la media (17.5), el $46.53 \%$ obtuvo una puntuación menor a la media (17.5) y el 5.61 \% tuvo una puntuación igual a la media (17.5).

\section{Discusión}

Las características sociodemográficas, definidas dentro de la población de estudio fueron: rango etario (18-35 años con $60.39 \%$, 36-59 años con $37.29 \%$ y 60 años o más con $2.31 \%$ ), género (femenino $82.83 \%$ y masculino $17.16 \%$ ), estado civil (soltero $56.76 \%$, casado 31.35 $\%, 6.27 \%$ divorciado y el $1.32 \%$ viudo) y lugar de residencia, destacando en los primeros cinco lugares los departamentos de Quetzaltenango con $28.05 \%$, Jutiapa con $18.81 \%$, Zacapa con $17.82 \%$, Guatemala con $17.16 \%$ y Totonicapán con $3.63 \%$ ).

Los medios de divulgación que más frecuentan los profesionales de la salud mental para mantenerse informados de la pandemia COVID-19, son las redes sociales con un $68.31 \%$, Identificando Facebook con un 72.27 \%, como la red social que más utilizan.

Comparando estos datos con un estudio realizado sobre la situación ocurrida durante el confinamiento en Paraguay, en referencia a la información divulgada por la pandemia COVID-19, Moreno-Fleitas, O. E. (2020) público unos datos compartidos por el Ministerio de Tecnologías de la Información y la Comunicación de Paraguay, donde se menciona que las redes sociales presentan un $71,6 \%$ de los medios de comunicación preferidos para informarse sobre actualizaciones del tema, destacando de un $10 \%$ un $9,5 \%$ la preferencia sobre la red social de Facebook. 
En su estudio, destaca que la mayoría de las noticias que se publican y circulan en el país no están basadas en fuentes científicas primarias, también, que los medios de comunicación social venden su propio espacio y que la información compartida puede ser real o errónea, influyendo en las opiniones sociales, por lo que es importante verificar la información que se recibe y concientizarse del medio que se obtiene. Los componentes del miedo que se presentaron en los profesionales fueron: activación somática con un $89.10 \%$, proceso cognitivo con un $58.74 \%$ y manifestaciones conductuales con un $54.45 \%$.

El miedo al coronavirus, en profesionales de la salud mental, se evidenció mediante la escala (FCV-19S), con un $90 \%$ de los casos igual o mayor a una puntuación de 17.5. Haciendo una comparación de este resultado con los obtenidos del artículo de Navarrete y colaboradores (2021) realizado con internos de enfermería y pre-profesionales en Ecuador, donde también se utilizó la escala FCV-19S, la presencia de miedo en profesionales de la salud es evidente. En el estudio se determinó que la muestra en general, presentó puntuaciones altas de miedo al COVID-19, destacando aún más las puntuaciones de los internos que estaban en la etapa inicial de la pandemia, adjudicándolo a la poca experiencia que tenían con posibles complicaciones y contagios de esta enfermedad infecciosa, por lo que, dentro de sus recomendaciones mencionan cuidar la salud de los profesionales y pre-profesionales que prestan sus servicios durante la COVID-19, y que los hallazgos relacionados con el estudio, influyan en esta iniciativa para intervenir y mejorar la salud mental de los profesionales de la salud, que también son vulnerables ante el coronavirus.

\section{Referencias}

Aleixandre-Benavent, R., Castelló-Cogollos, L., \& Valderrama-Zurián, J. C. (2020). Información y comunicación durante los primeros meses de Covid-19. Infodemia, desinformación y papel de los profesionales de la información. El profesional de la información (EPI), 29(4). DOI: https://doi.org/10.3145/epi.2020.jul.08

García-Marín, D. (2020). Infodemia global. Desórdenes informativos, narrativas fake y fact-checking en la crisis de la Covid-19//Global infodemic: Information disorders, false narratives, and fact checking during the Covid-19 crisis. Profesional de la información, 29(4). DOI: https://doi.org/10.3145/epi.2020.jul.11

Huarcaya-Victoria, J., Villarreal-Zegarra, D., Podestà, A. y Luna-Cuadros, M. A. (2020) Propiedades psicométricas de una versión española de la escala de miedo al COVID-19 en población general de Lima, Perú. Revista Internacional de Salud Mental y Adicciones (2020). DOI: https://doi.org/10.1007/s11469-020-00354-5

Lozano-Vargas, A. (2020). Impacto de la epidemia del Coronavirus (COVID-19) en la salud mental del personal de salud y en la población general de China. Revista de Neuro-Psiquiatría, 83(1), 51-56. DOI: https://doi.org/10.20453/rnp.v83i1.3687 
Mejía, C. R., Rodriguez-Alarcon, J. F., Garay-Rios, L., Enriquez-Anco, M. D. G., Moreno, A., Huaytan-Rojas, K., ... \& Curioso, W. H. (2020). Percepción de miedo o exageración que transmiten los medios de comunicación en la población peruana durante la pandemia de la COVID-19. Revista cubana de investigaciones biomédicas, 39(2). http://scielo.sld.cu/ pdf/ibi/v39n2/1561-3011-ibi-39-02-e698.pdf

Moreno-Fleitas, O. E. (2020). La divulgación de la información en la encrucijada de la crisis del COVID-19 en Paraguay. Reacciones y trasmisión de datos falsos y científicos a través de las redes sociales y los medios masivos. Rev. Soc. cient. Parag, 25(1), 58-85. DOI: https://doi.org/10.32480/rscp.2020-25-1.58-85

Navarrete, M. J. M., Cano, I. C. M., Suárez, P. C. M., \& Coronel, A. A. R. (2021). Impacto psicológico frente al COVID-19 en los internos de enfermería de prácticas pre-profesionales. Universidad Ciencia y Tecnología, 25(109), 53-59. DOI: https://doi.org/10.47460/uct.v25i109.448

Quezada, V. E. (2020). Miedo y psicopatología la amenaza que oculta el Covid-19. Cuadernos de Neuropsicología, 14(1), 19-23. file:///C:/Users/Myra\%20Santos/Downloads/Dialnet-MiedoYPsicopatologiaLaAmenazaQueOcultaEICovid19-7365556\%20(4).pdf

Ramírez-Coronel, A. A., Martínez-Suárez, P. C., Pogyo-Morocho, G. L., de los Ángeles Estrella-González, M., Mesa-Cano, I. C., Minchala-Urgilés, R. E., ... \& Abad-Martí, N. I. (2020). Evaluación psicométrica e intervención de enfermería frente al miedo a COVID-19. Archivos Venezolanos de Farmacología y Terapéutica, 39(5), 660-669. https://www.redalyc.org/ jatsRepo/559/55965386022/55965386022.pdf

Vera, C. A. R. (2020). Miedo a la COVID-19 en adultos peruanos mediante análisis de red. Revista Cubana de Enfermería, Revista Cubana de Enfermería. 2020; Vol. 36:e http://www. revenfermeria.sld.cu/index.php/enf/article/view/4082/664

\section{Sobre la autora}

\section{Luz Andrea Castañeda Argueta}

Tiene cierre de pensum de Licenciatura en Psicología en el Centro Universitario de Zacapa -CUNZAC-, actualmente estudiante de la Maestría en Neurociencias con énfasis en Neurocognición.

\section{Financiamiento de la investigación}

Con recursos propios de la investigadora.

\section{Declaración de intereses}

Declara no tener ningún conflicto de intereses, que puedan haber influido en los resultados obtenidos o las interpretaciones propuestas. 
Declaración de consentimiento informado

El estudio se realizó respetando el Código de ética y buenas prácticas editoriales de publicación.

Derechos de uso

Copyright@ 2022 por Luz Andrea Castañeda Argueta

Este texto está protegido por la Licencia Creative Commons Atribución 4.0 Internacional.

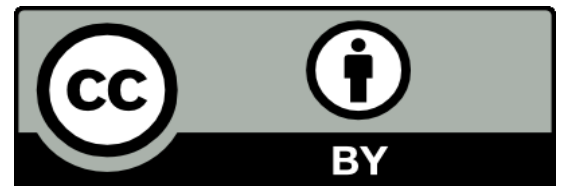

Este texto está protegido por una licencia

Creative Commons 4.0.

Es libre para compartir, copiar y redistribuir el material en cualquier medio o formato y adaptar el documento, remezclar, transformar y crear a partir del material para cualquier propósito, incluso comercialmente, siempre que cumpla la condición de atribución: debe reconocer el crédito de una obra de manera adecuada, proporcionar un enlace a la licencia, e indicar si se han realizado cambios. Puede hacerlo en cualquier forma razonable, pero no de forma tal que sugiera que tiene el apoyo del licenciante o lo recibe por el uso que hace. 